\title{
A case of missing identity
}

\author{
ABR Thomson MD FRCPC \\ "Tis a wise man who knows his father" \\ ABR Thomson
}

C ase reports are intended to stimulate, to provoke and to remind us to focus periodically on what we mean, or at least intend to mean. The accompanying paper by Chen and coauthors (pages 29-34) is a case - or three cases - in point. One patient initially received a diagnosis of ulcerative proctitis, which was later changed to Crohn's disease of the colon. Two patients had ileal Crohn's disease of the ileum, which was resected, and the inflammatory process recurred with a pattern reminiscent of classical ulcerative colitis, except that the patients had previously had Crohn's disease. The trio of authors reflect upon the literature, limited as it is, of six persons previously caught in this diagnostic challenge and reversal. Three patients were initially thought to have Crohn's disease of the ileum or ileum plus colon; ulcerative colitis was diagnosed 13 months to seven years later. In three patients, the initial diagnosis was distal colitis; terminal ileal disease compatible with Crohn's disease was diagnosed eight to 46 years later.

Now, this is not the simple phenomenon that we have all experienced - making the diagnosis of colonic inflammation most compatible with what we know as ulcerative colitis, and then over time, when a granuloma appears on biopsy or ileal inflammation develops, we change our minds, confessing that we were wrong the first time, that they really had Crohn's colitis and we had made a mistake. We all are prepared to accept the infallibility of our clinical, radiological, endoscopic and pathological acumen. We all frequently (the literature quotes $10 \%$ of the time) make the diagnosis of 'indeterminate colitis' and peddle water for a bit - and with the passage of time the correct diagnosis becomes more clear. But consider the possibility that the diagnosis was correct the first time - that the initially diagnosed ulcerative colitis did not 'turn into' Crohn's disease, but rather the two conditions truly coexisted in the same person.

Rather than picking apart these six previous cases, and the three that Chen et al now add to the world literature thanks to The Canadian Journal of Gastroenterology, let us pause and ask ourselves, "What is 'Crohn's disease'?" and "What is 'ulcerative colitis'?" Yes, these are chronic inflammatory disorders with no known, or at least proven, etiology, representing usually characteristic syndromes with extraintestinal and nutritional complications that occur with varying patterns, and are more or less treated with the same medications. But we are a long way from being able to quote line and verse of the specific immunological, genetic and environmental factors that are thought to be so important in the etiology of these two inflammatory conditions. Tests for the measurement of perinuclear antineutrophil cytoplasmic antibodies and anti-Saccharomyces cerevisiae antibodies are readily available from Prometheus Inc (San Diego, California) for all who can put their money on the

Department of Medicine, Division of Gastroenterology, University of Alberta, Edmonton, Alberta

Correspondence: Dr Alan BR Thomson, Department of Medicine, Division of Gastroenterology, 519 Newton Research Building, University of Alberta, Edmonton, Alberta T6G 2C2. Telephone 780-407-6490, fax 780-407-7964, e-mail alan.thomson@ualberta.ca 
barrelhead, but as Chen et al remark, "...the overall utility of these tests in clinical practice has yet to be determined", and two of their three cases illustrate the potential limitations of these serological tests when they are most needed to make a distinction. With the recognition of the disease activity-associated, enhanced intestinal permeability in patients with Crohn's disease, and more importantly findings of the $\mathrm{NOD}_{2}$ gene in about $30 \%$ of patients with typical Crohn's disease, recently reported in Nature by two separate groups $(1,2)$, we may be closer than we think to determining not only whether ulcerative colitis and Crohn's disease really are 'one or two separate conditions', but also, more importantly, their cause. The brave new world of pharmacogenetics will soon be within our reach, and we will be able to tailor specific therapy for individual patients.

So what have these three cases taught us? They have taught us to caution patients that we do not know the cause(s) of Crohn's disease or ulcerative colitis and that all the evidence available to clinicians supports one or the other diagnoses - but that the diagnosis may change, or be changed, but the treatment that we recommend is based on the best information that we have today. But tomorrow may be a better day, and we may be able to understand better why only some patients with ulcerative proctitis have their disease extend into pancreatitis, or why some patients with Crohn's disease have the inflammatory or stricturing form. So let us use these three cases to remind ourselves of what we do not know, and where future research will take us to care for our patients better.

\section{REFERENCES}

1. Hugot JP, Chamaillard M, Zouali H, et al. Association of NOD2 leucine-rich repeat variant with susceptibility to Crohn's disease. Nature 2001;411:599-603.

2. Ogura Y, Bonen DK, Inohara N, et al. A frameshift mutation in NOD2 associated with susceptibility to Crohn's disease. Nature 2001;411:603-6. 


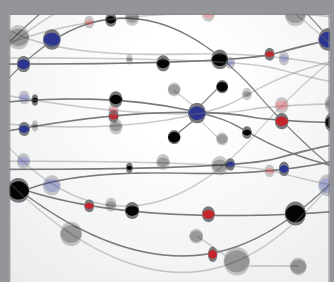

The Scientific World Journal
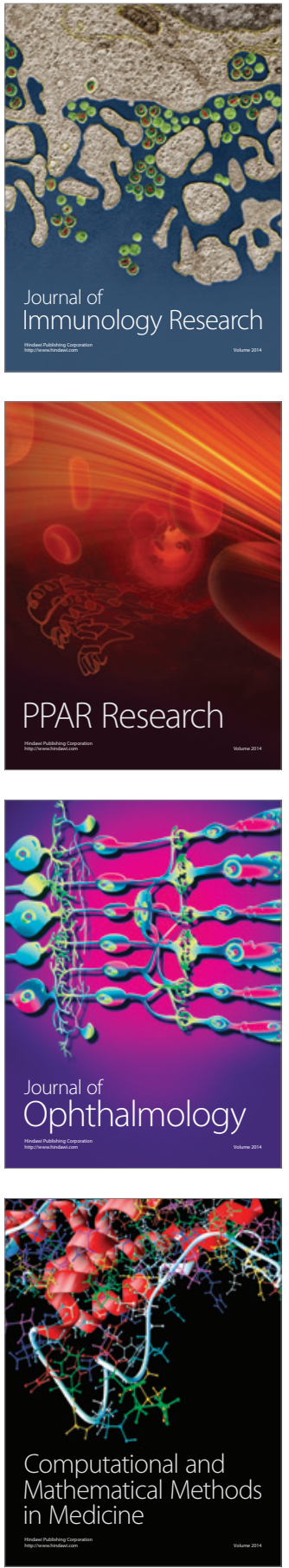

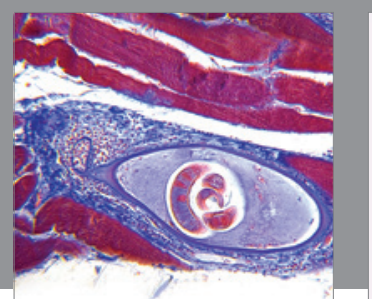

Gastroenterology Research and Practice

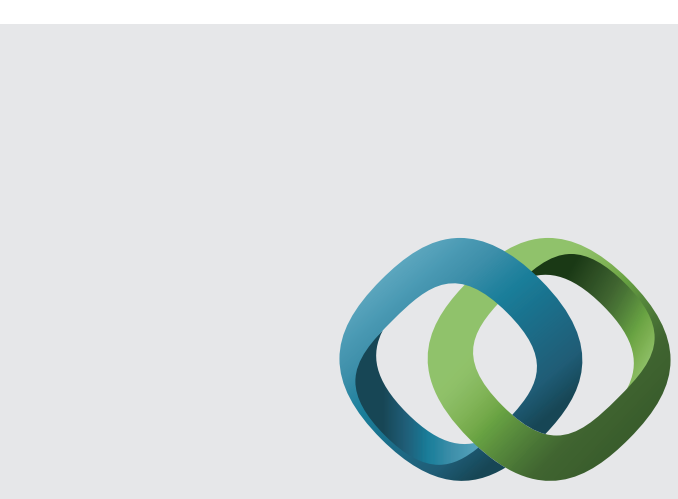

\section{Hindawi}

Submit your manuscripts at

http://www.hindawi.com
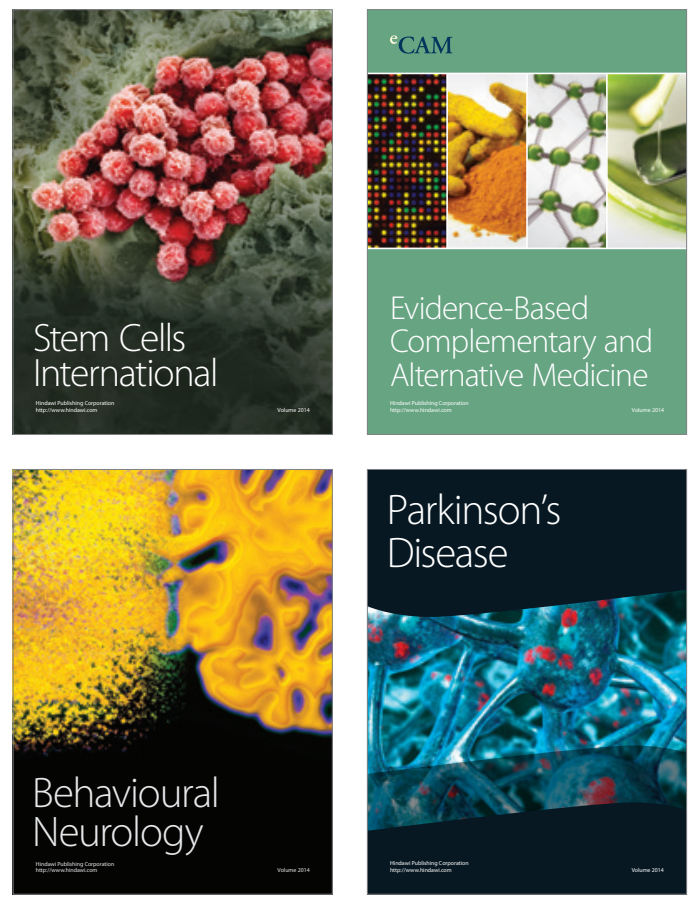
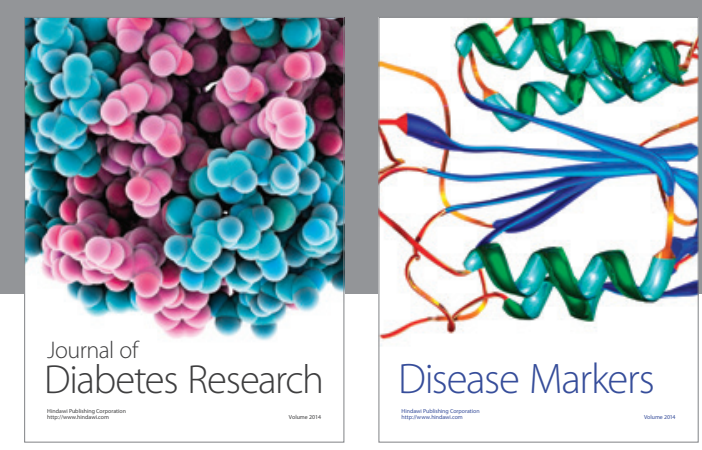

Disease Markers
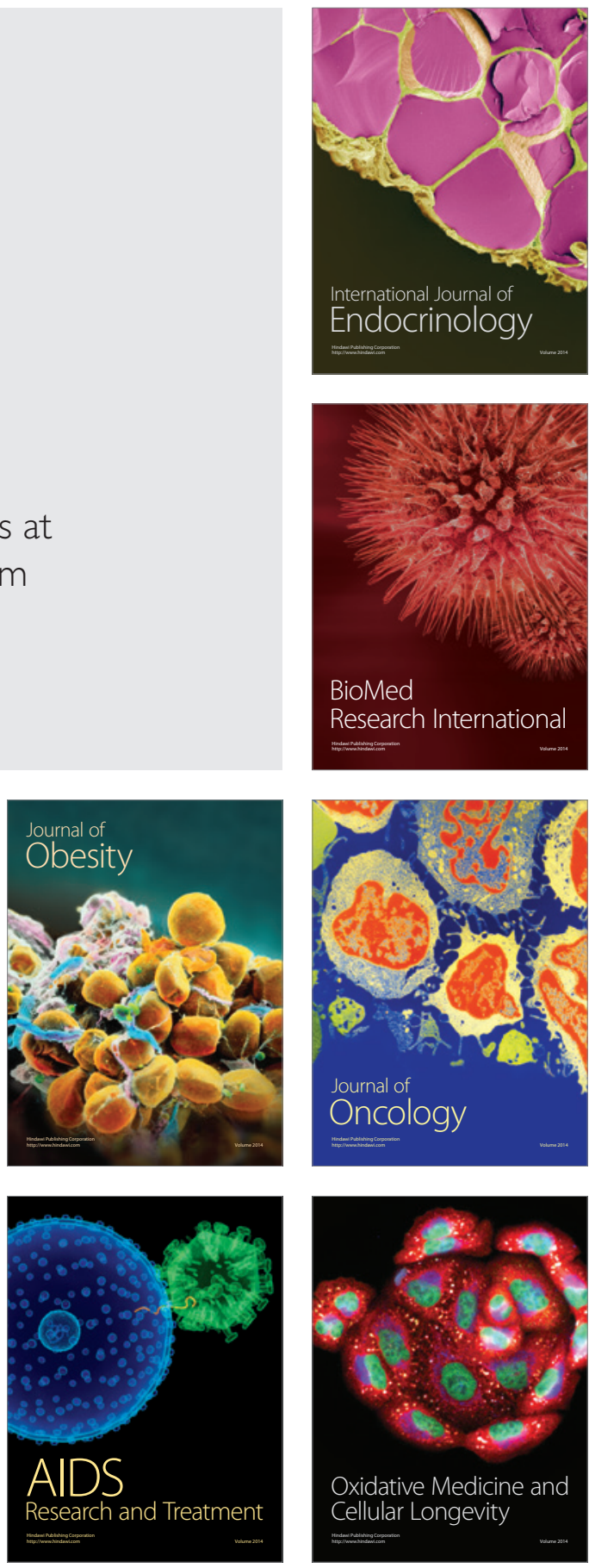